\title{
Intravenous immunoglobulin inhibits anti-glycoprotein Ilb-induced platelet apoptosis in a murine model of immune thrombocytopenia
}

\author{
Valery Leytin, ${ }^{1,2,3}$ Sergiy Mykhaylov, ${ }^{1}$ \\ Alison F. Starkey, ${ }^{1,4}$ David J. Allen, ${ }^{1}$ \\ Herbert Lau, ${ }^{1,3}$ Heyu Ni, ${ }^{1,2,3,4}$ John W. \\ Semple, ${ }^{1,2,4,5}$ Alan H. Lazarus ${ }^{1,2,4,6}$ and \\ John Freedman 1,2,3,6 \\ ${ }^{1}$ Division of Transfusion Medicine, Department of \\ Laboratory Medicine, St Michael's Hospital, \\ ${ }^{2}$ Toronto-Hamilton Platelet Immunobiology \\ Group, ${ }^{3}$ Department of Laboratory Medicine and \\ Pathobiology, University of Toronto, ${ }^{4}$ The \\ Canadian Blood Services, ${ }^{5}$ Department of \\ Pharmacology, University of Toronto, and \\ ${ }^{6}$ Department of Medicine, University of Toronto, \\ Toronto, ON, Canada
}

\begin{abstract}
Summary
We have previously shown that injection of anti-glycoprotein (GP) IIb induces murine immune thrombocytopenia (ITP) and that intravenous immunoglobulin (IVIg) ameliorates ITP. We hypothesise that murine ITP may be associated with platelet apoptosis, which is upregulated by anti-GPIIb and downregulated by IVIg. The current study demonstrated that anti-GPIIb injection induced three critical apoptosis manifestations in platelets: (i) mitochondrial inner transmembrane potential $(\Delta \Psi \mathrm{m})$ depolarisation; (ii) caspase-3 activation; and (iii) phosphatidylserine (PS) exposure. IVIg administration inhibited caspase-3 activation and PS exposure, but not $\Delta \Psi \mathrm{m}$-depolarisation, in anti-GPIIb-treated platelets, demonstrating that IVIg ameliorates thrombocytopenia concomitantly with inhibiting late, but not early mechanisms of platelet apoptosis.
\end{abstract}

Keywords: murine model, immune thrombocytopenia, anti-glycoprotein IIb antibody, intravenous immunoglobulin, platelet apoptosis.

Received 3 November 2005; accepted for

publication 2 December 2005

Correspondence: Dr Valery Leytin, Transfusion

Medicine, St Michael's Hospital, 30 Bond St,

Toronto, ON, Canada M5B 1W8.

E-mail: leytinv@smh.toronto.on.ca

While intravenous immunoglobulin (IVIg) is used to treat immune thrombocytopenia (ITP) and other autoimmune diseases, its mechanism of action remain unclear. Several theories have been proposed, including the ability of IVIg to block the reticuloendothelial system (RES), an effect on the inhibitory Fc $\gamma$ RIIB receptor, neutralisation by anti-idiotypic antibodies and modulation of cytokine cascades (Lazarus \& Crow, 2003). An effect of IVIg on apoptosis of nucleated cells has been demonstrated both in vivo and in vitro; IVIg manifests anti-apoptotic or pro-apoptotic action depending on the targeted cell type (Prasad et al, 1998; Viard et al, 1998).

Over the last decade, apoptosis of anucleated cytoplasts and platelets has been recognised (Vanags et al, 1997; Wolf et al, 1999; Brown et al, 2000; Leytin \& Freedman, 2003; Leytin et al, 2004). Platelet apoptosis has been documented in different models using well-defined apoptosis markers of nucleated cells (Budihardjo et al, 1999; Kroemer \& Reed, 2000) such as depolarisation of the mitochondrial inner transmembrane potential $(\Delta \Psi \mathrm{m}), \mathrm{Bcl}-2$ family proteins expression, caspase-3 activation and phosphatidylserine (PS) exposure (Leytin \& Freedman, 2003). These data demonstrate that platelets contain the essential components for cytoplasmic apoptosis.

Many antiplatelet antibodies, notably anti-glycoprotein (GP) IIbIIIa and anti-GPIb, induce ITP in humans and mice (Nieswandt et al, 2000). Some of these antibodies induce thrombocytopenia by targeting platelets through Fc receptors in the RES, while others act by Fc-independent mechanisms (Nieswandt et al, 2000). We have previously shown that profound thrombocytopenia induced in mice by injection of antiplatelet antibodies can be ameliorated by IVIg treatment 
(Crow et al, 2001; Song et al, 2003; Siragam et al, 2005). Piguet and Vesin (2002) reported that antiplatelet antibodies induced thrombocytopenia in murine models; this was associated with activation of platelet caspases and prevented by treatment with a pan-caspase inhibitor ZVAD-fmk. In contrast, injection of antagonistic anti-apoptotic antibodies increased platelet count and lifespan due to decreased caspase activation (Piguet \& Vesin, 2002). Taken together, these data suggest a possible causal role of platelet apoptosis in the development of thrombocytopenia, which can be induced by pro-apoptotic antibodies and prevented by apoptosis inhibitors and anti-apoptotic antibodies.

The present study tested the hypothesis that injection of anti-GPIIb antibody will induce apoptosis in mouse platelets and that treatment with IVIg will inhibit platelet apoptosis thus potentially ameliorating ITP.

\section{Materials and methods}

C57BL/6 mice were purchased from The Jackson Laboratory (Bar Harbor, ME, USA) and treated as described in Fig 1. Blood from saphenous veins was collected into capillary tubes and $100 \mu \mathrm{l}$ of blood was diluted in $900 \mu \mathrm{l}$ of buffer A [ $145 \mathrm{mmol} / \mathrm{l} \mathrm{NaCl}, 5 \mathrm{mmol} / \mathrm{l} \mathrm{KCl}, 1 \mathrm{mmol} / \mathrm{l} \quad \mathrm{MgCl}_{2}$, $5.6 \mathrm{mmol} / \mathrm{l}$ glucose, $0 \cdot 1 \%$ bovine serum albumin (BSA), $10 \mathrm{mmol} / \mathrm{l}$ HEPES, pH 7·4], containing $0 \cdot 42 \%$ sodium citrate.

The platelet count was determined in blood diluted (1:10) with buffer A, containing sodium citrate using a Coulter LH750 Analyzer (Beckman-Coulter, Miami, FL, USA). Platelet-rich plasma (PRP) was prepared by centrifugation of the 1:10 diluted blood for $5 \mathrm{~min}$ at $209 \mathrm{~g}$ for determining platelet apoptosis by flow cytometry (Leytin et al, 2004).

Depolarisation of $\Delta \Psi \mathrm{m}$ was determined using the cellpenetrating lipophilic cationic fluorochrome JC-1 (Molecular Probes, Eugene, OR, USA) which accumulates in the mitochondrial matrix, driven by $\Delta \Psi \mathrm{m}$ (Kroemer \& Reed, 2000), and expressed as an increase of green-to-red (G/R) fluorescent ratio reflecting the decrease in content of JC-1 aggregates when the inner mitochondrial membrane becomes depolarised (Leytin et al, 2004). The JC-1 stock solution $[2 \mathrm{mg} / \mathrm{ml}$ in dimethylsulphoxide (DMSO); Sigma Chemical Co., St Louis, MO, USA) was diluted with buffer B [phosphate-buffered saline (PBS; Sigma) supplemented with $1 \mathrm{mmol} / 1 \mathrm{MgCl}_{2}$, $5.6 \mathrm{mmol} / \mathrm{l}$ glucose, $0 \cdot 1 \% \mathrm{BSA}, 10 \mathrm{mmol} / \mathrm{l}$ HEPES, $\mathrm{pH} 7 \cdot 4$ ] to a final concentration of $1 \mu \mathrm{g} / \mathrm{ml}$, mixed by vortex, incubated in the dark for $15 \mathrm{~min}$ at room temperature and centrifuged for $15 \mathrm{~min}$ at $5996 \mathrm{~g}$ in $1.5 \mathrm{ml}$ eppendorf tubes in an Eppendorf 5415C microfuge (Eppendorf, Westbury, NY, USA). The supernatants were harvested, combined and used immediately in the $\Delta \Psi \mathrm{m}$ depolarisation assay as the JC-1 working solution. Aliquots $(40 \mu \mathrm{l})$ of PRP were incubated in the dark for $45 \mathrm{~min}$ at $37^{\circ} \mathrm{C}$ with $460 \mu \mathrm{l}$ of JC- 1 working solution and samples were acquired on a FACScan flow cytometer (BD Biosciences, San Jose, CA, USA).
Caspase- 3 activation was measured by the cell-penetrating carboxyfluorescein-labelled fluoromethyl ketone tetrapeptide (FAM-DEVD-FMK; Chemicon International, Temecula, CA, USA) that specifically and covalently binds to active caspase-3 (Piguet \& Vesin, 2002; Leytin et al, 2004). FAM-DEVD-FMK was dissolved in $50 \mu \mathrm{l}$ of DMSO to create a $150 \mathrm{x}$ stock solution, according to the manufacturer's recommendation, diluted (1:24) with PBS, pH 7·4, mixed by vortex and used immediately in caspase- 3 activation assay. Aliquots $(40 \mu \mathrm{l})$ of PRP were incubated in the dark for $1 \mathrm{~h}$ at $37^{\circ} \mathrm{C}$ with $10 \mu \mathrm{l}$ of the 1:24 diluted FAM-DEVD-FMK solution, $450 \mu$ of buffer A was added and samples were analysed by flow cytometry.

Phosphatidylserine exposure on the platelet surface was determined by dual colour assay using concurrent staining of platelets with fluorescein isothiocyanate (FITC)-conjugated annexin $\mathrm{V}$ and phycoerythrin (PE)-conjugated hamster antimouse CD61 monoclonal antibody (BD Biosciences Pharmingen, San Diego, CA, USA). Aliquots $(30 \mu \mathrm{l})$ of PRP, further diluted (1:6) with buffer A, were mixed with $10 \mu \mathrm{l}$ of buffer A containing $8 \mathrm{mmol} / \mathrm{l}$ of $\mathrm{CaCl}_{2}$ and incubated in the dark for $15 \mathrm{~min}$ at room temperature with a mixture of $2.5 \mu \mathrm{l}$ annexin V-FITC and 2.5 $\mu \mathrm{l}$ anti-CD61-PE. Buffer A $(300 \mu \mathrm{l})$ containing $2 \mathrm{mmol} / \mathrm{l}$ of $\mathrm{CaCl}_{2}$ was then added and samples were analysed by flow cytometry using fluorescence- 2 threshold to acquire platelet (CD61)-positive events (Leytin et al, 2004).

\section{Results and discussion}

Figure 1A shows that injection of anti-mouse GPIIb antibody induced marked thrombocytopenia in C57BL/6 mice, resulting in a drop of peripheral platelet count to $23.4 \pm 1.9 \%$ of the mean value in untreated animals $(P<0.0001)$. Administration of non-immune IgG (used as a negative control for anti-GPIIb) did not induce thrombocytopenia. However, injection of $50 \mathrm{mg}$ IVIg per mouse (equivalent to a dose of $2 \mathrm{~g} / \mathrm{kg}$ body weight, used for ITP treatment in humans) significantly ameliorated anti-GPIIb-induced thrombocytopenia $(P<0.0001)$.

After injection of anti-GPIIb, platelets showed significantly higher caspase-3 activation (Fig 1B, $P=0.02$ ), PS exposure (Fig $1 \mathrm{C}, P=0.04$ ) and $\Delta \Psi \mathrm{m}$ depolarisation (Fig 2, $P=0.02$ ). Treatment with IVIg ameliorated the anti-GPIIb-induced caspase- 3 activation $(P=0.04)$ and PS exposure $(P=0.03)$ in the platelets (Fig $1 \mathrm{~B}$ and $\mathrm{C}$ ), but did not inhibit $\Delta \Psi \mathrm{m}$ depolarisation (Fig 2, $P=0 \cdot 42$ ).

Two main pathways have been shown to lead to apoptosis in nucleated cells. The first, is the extrinsic pathway, which is initiated via the cell-surface death receptors, while the second is the intrinsic pathway, which is triggered by disruption of mitochondrial integrity including $\Delta \Psi \mathrm{m}$ depolarisation. Activation of executioner caspase- 3 and PS exposure are downstream of the extrinsic death signalling and intrinsic mitochondrial disintegration pathways (Budihardjo et al, 1999; Kroemer \& Reed, 2000; Leytin \& Freedman, 2003). Aberrant exposure of PS residues on the outer plasma 
(A)

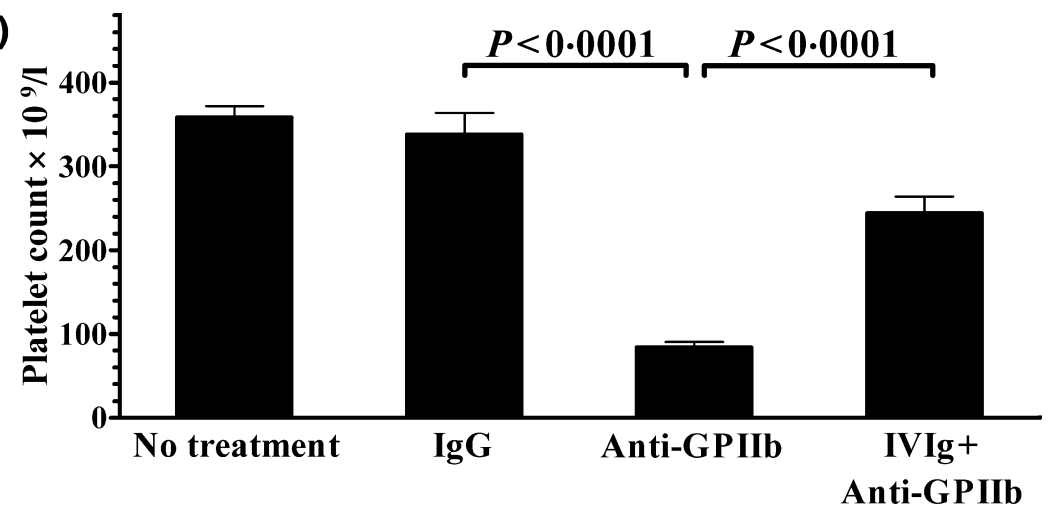

(B)
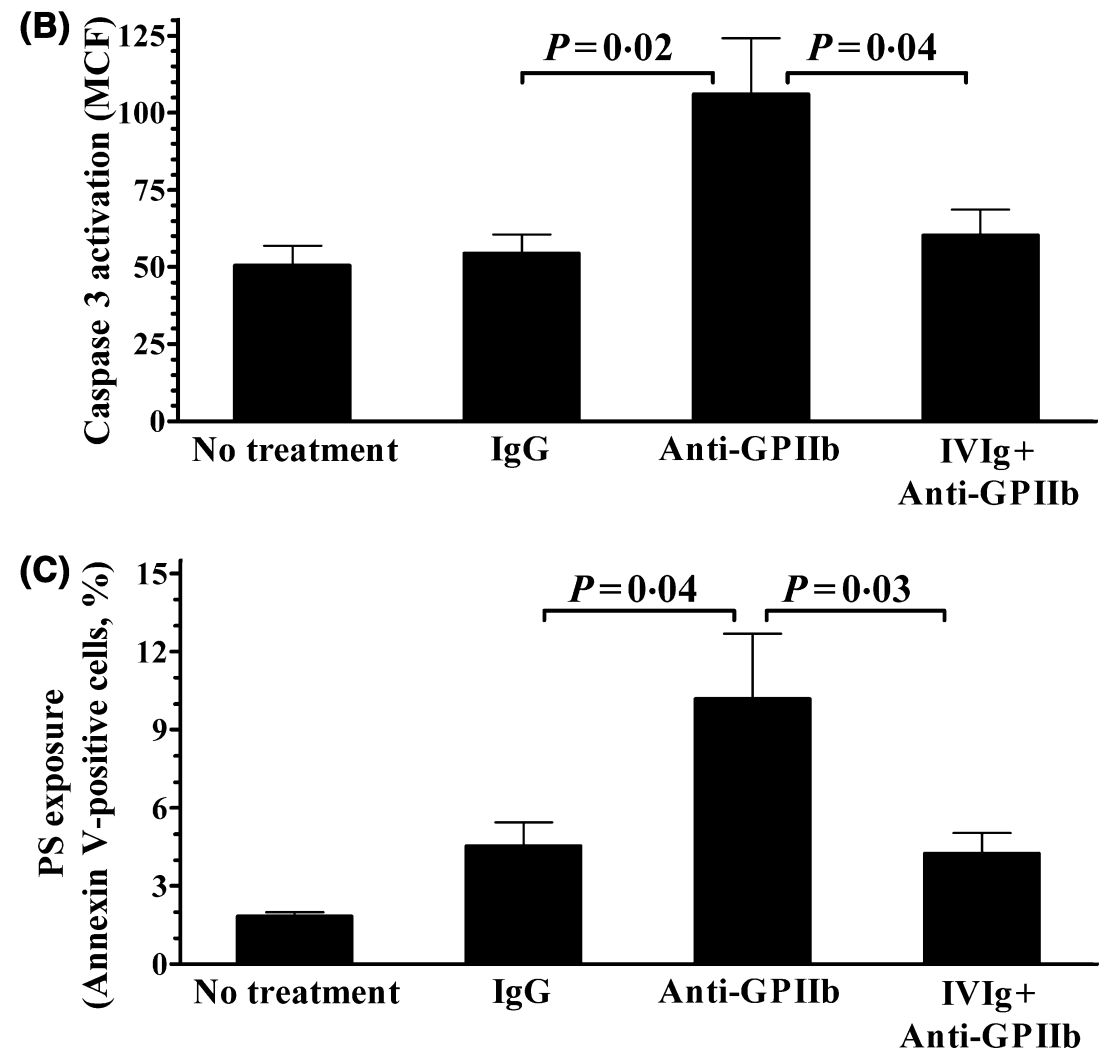

Fig 1. Administration of intravenous immunoglobulin (IVIg) ameliorates anti-glycoprotein (GP) IIb-induced immune thrombocytopenia (A), and inhibits caspase-3 activation (B) and phosphatidylserine (PS) exposure (C) in mouse platelets. In the anti-GPIIb-treatment group, thrombocytopenia was induced on day 1 by intraperitoneal injection of $2 \mu \mathrm{g}$ rat anti-mouse GPIIb (MWReg30, IgG1; PharMingen, Mississauga, ON, Canada) in $200 \mu 1$ of phosphate-buffered saline ( $\mathrm{pH} 7 \cdot 2$ ). In the control IgG-treatment group, $2 \mu \mathrm{g}$ of non-immune rat IgG was injected on day 1 . In the IVIg + antiGPIIb-treatment group, mice were injected intraperitoneally on day 0 with $500 \mu 1$ of $10 \%$ IVIg $(50 \mathrm{mg} / \mathrm{mice}, 2 \mathrm{~g} / \mathrm{kg}$; Gamimune $10 \%$; Bayer, Elkhart, IN, USA), followed by injection of $2 \mu \mathrm{g}$ anti-GPIIb on day 1 . Platelet enumerations and apoptosis tests were assayed on day 2 . In the 'no treatment' group, mice were bled before administration of IgG, anti-GPIIb or IVIG + anti-GPIIb, and platelet count and apoptosis were analysed on day 0 . Platelet caspase-3 activation was quantified as the mean channel fluorescence (MCF) of FAM-DEVD-FMK-labelled platelets. Platelet PS exposure was determined by annexin V-binding assay as the percentage of annexin V-positive cells. Results of three independent experiments, each using two mice per group, are presented. Hence, mean \pm SEM for six mice per group are shown and $P$-values between indicated groups were calculated by unpaired $t$-test; for 'no treatment' versus IgG-treatment groups, $P=0.51$ (A), $P=0.67$ (B) and $P=0.04$ (C).

membrane leaflet of nucleated cells allows for the phagocytic recognition and elimination of apoptotic cells (Budihardjo et al, 1999; Kroemer \& Reed, 2000; Leytin \& Freedman, 2003). In human platelets stimulated with calcium ionophore A23187, caspase-3 activation and PS exposure are also downstream of $\Delta \Psi \mathrm{m}$ depolarisation (Leytin et al, 2004). The data presented in this study indicate that IVIg does not affect upstream breakdown of $\Delta \Psi \mathrm{m}$ in platelets (Fig 2), but does inhibit downstream apoptotic events, caspase-3 activation (Fig 1B) and PS exposure (Fig 1C). 

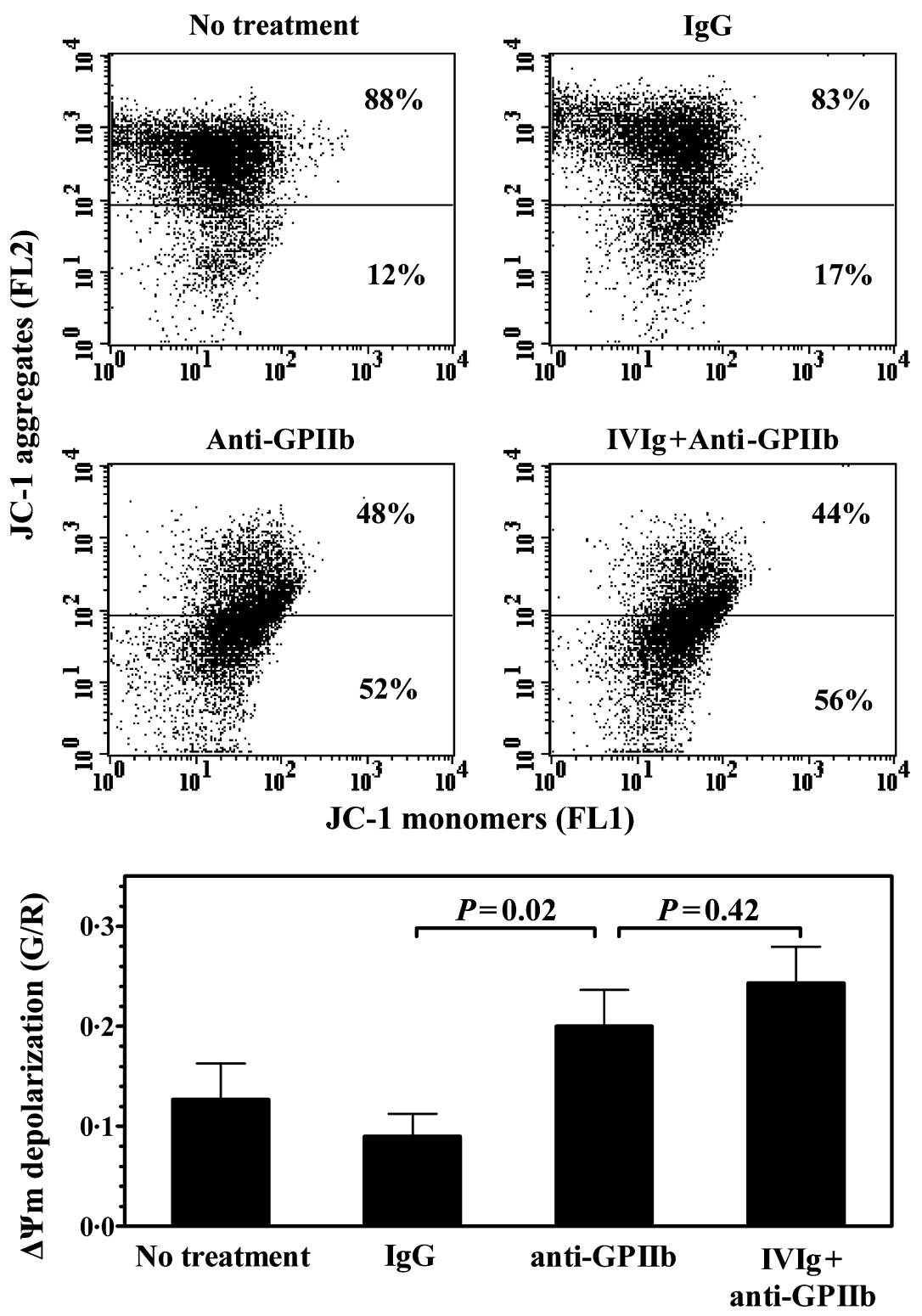

Fig 2. Administration of anti-glycoprotein (GP) IIb induces depolarisation of mitochondrial inner transmembrane potential ( $\Delta \Psi \mathrm{m})$ in mouse platelets, but intravenous immunoglobulin (IVIg) does not inhibit $\Delta \Psi \mathrm{m}$ depolarisation. Mice were either untreated or injected with non-immune IgG, anti-GPIIb or IVIg + anti-GPIIb as described in Fig 1. Platelet $\Delta \Psi \mathrm{m}$ depolarisation was determined using the JC-1 fluorescent probe. In the upper and middle panels, representative flow cytometric dot plots are shown and percentages of platelets containing high or low levels of JC-1 aggregates presented. In the lower panel, depolarisation of platelet $\Delta \Psi \mathrm{m}$ was quantified as the green-to-red (G/R) fluorescence ratio. Results of three independent experiments, each using two mice per group, are presented. Hence, mean \pm SEM for six mice per group are shown and $P$-values between indicated groups were calculated by unpaired $t$-test; for 'no treatment' versus IgG-treatment groups, $P=0 \cdot 41$.

Nieswandt et al (2000) demonstrated that the specificity of the target antigen recognised by antiplatelet antibodies determined the mechanism of phagocytic clearance of platelets in murine ITP. They have shown that ITP is induced by injection of intact anti-GPIIbIIIa monoclonal antibodies, but not $\mathrm{F}(\mathrm{ab})_{2}$ fragments, indicating $\mathrm{Fc}$-dependent mechanism of platelet phagocytosis provoked by anti-GPIIbIIIa. In contrast, ITP induced by anti-GPIb $\alpha$ monoclonal antibodies is Fc-independent. Although several mechanisms have been proposed to explain the success of IVIg therapeutic treatment in ITP, the most accepted mechanism is the competitive inhibition of $\mathrm{Fc}$ receptors on phagocytic cells (reviewed by Lazarus \& Crow, 2003). In a murine ITP model, we have shown that IVIg does not inhibit the binding of monoclonal anti-GPIIb to platelets both in vitro and in vivo (Crow et al, 2001). Taken together, these data suggest that: (i) platelet apoptosis induced by antiGPIIb is an Fc-receptor-mediated process, i.e. a process dependent on the interactions of $\mathrm{Fc}$ fragments of anti-GPIIb antibodies with $\mathrm{Fc}$ receptors on phagocytic cells; and (ii) the suppressive effect of IVIg on anti-GPIIb-induced downstream 
platelet apoptotic events is the result of inhibition of Fc fragment-Fc receptor interactions, rather than inhibition of anti-GPIIb binding to platelets.

In summary, we have shown that in vivo administration of an antibody against GPIIb is associated with apoptotic changes in mouse platelets characterised by the dissipation of mitochondrial transmembrane potential, activation of caspase-3 and exposure of PS. However, IVIg treatment significantly inhibited platelet caspase-3 activation and PS exposure. While the extent of the role played by apoptosis in the pathogenesis of ITP remains unknown, these results suggest a novel mechanism of IVIg action involving anti-apoptotic effects in platelets that may contribute to the amelioration of ITP.

\section{Acknowledgements}

This work was supported by a grant from the Bayer-Canadian Blood Services-Héma-Québec-CIHR Partnership Fund.

\section{References}

Brown, S.B., Clarke, M.C., Magowan, L., Sanderson, H. \& Savill, J. (2000) Constitutive death of platelets leading to scavenger receptormediated phagocytosis. A caspase-independent cell clearance program. Journal of Biological Chemistry, 275, 5987-5996.

Budihardjo, I., Oliver, H., Lutter, M., Luo, X. \& Wang, X. (1999) Biochemical pathways of caspase activation during apoptosis. Annual Review of Cell and Developmental Biology, 15, 269-290.

Crow, A.R., Song, S., Semple, J.W., Freedman, J. \& Lazarus, A.H. (2001) IVIg inhibits reticuloendothelial system function and ameliorates murine passive-immune thrombocytopenia independent of anti-idiotype reactivity. British Journal of Haematology, 115, 679686.

Kroemer, G. \& Reed, J.C. (2000) Mitochondrial control of cell death. Nature Medicine, 6, 513-519.

Lazarus, A.H. \& Crow, A.R. (2003) Mechanism of action of IVIG and anti-D in ITP. Transfusion and Apheresis Science, 28, 249-255.
Leytin, V. \& Freedman, J. (2003) Platelet apoptosis in stored platelet concentrates and other models. Transfusion and Apheresis Science, 28, 285-295.

Leytin, V., Allen, D.J., Mykhaylov, S., Mis, L., Lyubimov, E.V., Garvey, B. \& Freedman, J. (2004) Pathologic high shear stress induces apoptosis events in human platelets. Biochemical and Biophysical Research Communications, 320, 303-310.

Nieswandt, B., Bergmeier, W., Rackebrandt, K., Gessner, J.E. \& Zirngibl, H. (2000) Identification of critical antigen-specific mechanisms in the development of immune thrombocytopenic purpura in mice. Blood, 96, 2520-2527.

Piguet, P.F. \& Vesin, C. (2002) Modulation of platelet caspases and life-span by anti-platelet antibodies in mice. European Journal of Haematology, 68, 253-261.

Prasad, N.K., Papoff, G., Zeuner, A., Bonnin, E., Kazatchkine, M.D., Ruberti, G. \& Kaveri, S.V. (1998) Therapeutic preparations of normal polyspecific IgG (IVIg) induce apoptosis in human lymphocytes and monocytes: a novel mechanism of action of IVIg involving the Fas apoptotic pathway. Journal of Immunology, 161, 3781-3790.

Siragam, V., Brinc, D., Crow, A.R., Song, S., Freedman, J. \& Lazarus, A.H. (2005) Can antibodies with specificity for soluble antigens mimic the therapeutic effects of intravenous IgG in the treatment of autoimmune disease? Journal of Clinical Investigations, 115, 155-160.

Song, S., Crow, A.R., Freedman, J. \& Lazarus, A.H. (2003) Monoclonal IgG can ameliorate immune thrombocytopenia in a murine model of ITP: an alternative to IVIG. Blood, 101, 3708-3713.

Vanags, D.M., Orrenius, S. \& Aguilar-Santelises, M. (1997) Alterations in Bcl-2/Bax protein levels in platelets form part of an ionomycininduced process that resembles apoptosis. British Journal of Haematology, 99, 824-831.

Viard, I., Wehrli, P., Bullani, R., Schneider, P., Holler, N., Salomon, D., Hunziker, T., Saurat, J.H., Tschopp, J. \& French, L.E. (1998) Inhibition of toxic epidermal necrolysis by blockade of CD95 with human intravenous immunoglobulin. Science, 282, 490-493.

Wolf, B.B., Goldstein, J.C., Stennicke, H.R., Beere, H., AmaranteMendes, G.P., Salvesen, G.S. \& Green, D.R. (1999) Calpain functions in a caspase-independent manner to promote apoptosis-like events during platelet activation. Blood, 94, 1683-1692. 